\title{
Earth as a Gravitational-Wave Interferometr
}

\author{
Vadim Il'chenko ${ }^{1, *}$ \\ ${ }^{1}$ Geological Institute, Kola Science Center Russian Academy of Sciences, RU-184209, Apatity, Russia
}

\begin{abstract}
Based on the principle of Equivalence of Gravitating Masses (EGM) and tectonostratigraphic model of the Earth outer shell structure (the Earth crust and upper mantle), the average depth of the lunar mass gravitational influence on the Earth was calculated as $\sim 1600 \mathrm{~km}$. The developed model is based on the mechanism of rocks tectonic layering of the Earth crust-mantle shell as an oscillatory system with dynamic conditions of a standing wave, regularly excited by the lunar tide and immediately passing into the damping mode. After comparing the average depth of solid lunar tide impact of $\sim 1600 \mathrm{~km}$ with the height of the solid lunar tide "hump" on the Earth surface of $0.5 \mathrm{~m}$, a "tensile strain" was calculated with an amplitude only one order of magnitude larger than the amplitude of the gravitational wave recorded by the Advanced LIGO interferometer: $\mathrm{A} \approx 10-18 \mathrm{~m}$ (the merger result of a black holes pair ca $1.3 \mathrm{Ga}$ ago). The results of the present study suggest that the crust-mantle shell of the Earth may be used as a gravitational-wave interferometer.
\end{abstract}

\section{Introduction}

One of the 2017 Nobel Prizes was awarded for the creation of a gravity wave detector - an Advanced LIGO interferometer (the construction cost of which amounted to more than 600 million US dollars) and detection of a gravitational wave with an amplitude of $\mathrm{A}=10^{-18} \mathrm{~m}$. It is believed that this wave was initiated by the merger of two black holes $\approx 1.3$ billion years ago [1]. Assuming that the speed of the gravitational wave is equal to the speed of electromagnetic waves (light, etc.), then the source of this wave is located at a distance of $\mathrm{R} \approx 1.3$ billion light years $(\approx 400$ MParsecs) from the Earth. The existence of gravitational waves was predicted by Einstein in his general theory of relativity $\approx 100$ years ago. According to his theory, gravitational waves (perturbation, curvature of space-time) are created by any massive object moving with acceleration. The list of such objects includes, e.g., the Moon, moving in a nearearth elliptical orbit with variable $( \pm)$ acceleration. This movement of the moon regularly creates a pair of antipode waves of a solid tide in the outer Earth's shell, turning this shell into an oscillating system with dynamic conditions of a standing wave in the damping mode. As the oscillations damp, conditions for further tectonic stratification of the geological environment arise. The gravitational origin of the waves of the solid lunar tide in the outer shell of the Earth is quite obvious and does not need any special substantiation.

\section{Tectonic layering mechanism}

The mechanism of tectonic layering of the Earth's crustmantle shell (CMS) as a damping oscillatory system was established by studying the physical properties of the core of the Kola superdeep Borehole (SG-3) and modeling the geological and geophysical structure of the Earth's crust within the Pechenga block (Baltic Shield) [2].

Gravitational perturbation from the lunar mass $\left(\mathrm{M}_{\mathrm{L}}\right)$ turns the CMS into an oscillatory system, and the oscillations immediately starts damping in the mode of a standing wave, similar to the decaying sound of a vibrating string. This damping proceeds in a stepwise rhythm with the development of a system of fixed nodal points: the first node (excluding the nodes at the points of attachment of the string, the length of the string $L$ is equal to the length of the standing wave $-\lambda$ ) appears in the middle of the string, dividing this string in half, and the wave into two half-waves $(\lambda / 2)$; then, as energy is absorbed by oscillations, new nodes appear in the middle of each of these half-waves, etc. The damping proceeds (wave division with their multiplication in a step-by-step rhythm) according to the law:

$$
\lambda_{\mathrm{n}}=\lambda 0 / 2^{\mathrm{n}}
$$

(n - mode number: $0,1,2, \ldots$ all integers numbers, $\lambda_{n}$ is the length of the n-th mode or the distance between adjacent nodes, $\lambda 0$ is the total length of the oscillatory system or mode No. $0=\mathrm{M} 0$ ). The attenuation of sound from a string differs but little from the damping of a standing wave in CMS rocks. This process leads to the development of a system of fixed nodal points, to which some of the stresses from the oscillating antinode zones are "squeezed out". Over time, these nodes turn into concentrators of excessively high stresses, which is confirmed by experiments [3].

The spatial position of these nodes - concentrators of excessively high stresses in the outer shell of the Earth (here: in the earth's crust), is determined by the formula: 


$$
\mathrm{M}_{\mathrm{n}}=\mathrm{M} 0 / 2^{\mathrm{n}},\left(\mathrm{M} 0=\mathrm{M}_{\mathrm{n}} \cdot 2^{\mathrm{n}}\right. \text {, respectively) }
$$

(n - mode No.: 0, 1, 2, ... all integers, it is determined using a special table [2, 4]; $M_{n}$ is the length of the $n$-th mode - the distance between neighboring nodes of the same mode, the thickness of the CMS of the Earth or the earth's crust can be considered as No. $0=\mathrm{M} 0$ ). In the vicinity of nodal points (concentrators of excessively high stresses) the boreholes have increased diameter due to the inrush (break out) of rocks from the borehole walls. The system of nodes creates the prerequisites for the development of subhorizontal rock intervals with an excessively high stress state, potentially determining the development of tectonic boundaries in the CMS of the Earth.

Tectonic boundaries are, as a rule, distinguished by a rhythmic spatial distribution of the fractal type, which is easily recorded by borehole geophysics of borehole logging (logging plots of different types that reflect the dynamic state of rocks along the section). These features are used in tectonostratigraphic models of the structure of the CMS for the last ten years [4]. Models based on borehole logging data from shallow boreholes can be used to calculate the spatial position (depth) of rock intervals with excessively stressed state, which can occur below the bottom of these shallow boreholes (at almost any depth). Such rock bodies (stress concentrators), may cause such emergency situations as crush down of the mine roof, rocks break out from the walls of boreholes, etc., during mining operations - underground mining, drilling, etc. which may lead to the destruction of expensive equipment and human casualties.

\section{EGM principle}

In modern physics, in addition to Newton's theory, there are $\approx 26$ gravitational theories [5], which number raises doubts about the correctness of at least one of them. Hypothetically, gravitational interaction is carried out in the form of the exchange of massive objects by gravitons [6]. If this is indeed so, then the number of gravitons participating in the exchange between such celestial bodies as the Moon and the Earth should be equivalent to their masses $\left(\mathrm{M}_{\mathrm{L}}\right.$ and $\mathrm{M}_{\mathrm{E}}$, respectively). The idea of "graviton equivalence" was the reason for the following postulate.

The depth of penetration of lunar gravity into the bowels of the Earth is determined by the principle of Equivalence of Gravitational Masses (EGM) - not to be confused with the equivalence principle in Einstein's general relativity theory (!), since there is no equivalence between inertial and gravitational mass [7]. Our EGM principle states that the mass of matter in the region affected by the lunar tidal disturbance in the Earth's shell is equal to the mass of the disturbance source, i.e. - the Moon (where the number of gravitons is equivalent to the mass of source elementary particles of the Moon, whose mass $=M_{L}$ ), and the radius of the wave of the lunar tide wave $\mathrm{R}_{\mathrm{LTw}}$ in the Earth's shell is determined by the formula:

$$
\mathrm{R}_{\mathrm{LTW}}=\left(3 \mathrm{M}_{\mathrm{L}} / 4 \pi \rho_{\mathrm{CMES}}\right)^{1 / 3} \text {, }
$$

(3)

$\mathrm{ML}=7,3477 \times 1022 \mathrm{~kg}, \pi=3.14, \rho \mathrm{CMES} \approx 4.5 \mathrm{~g} / \mathrm{cm} 3-$ average density of the Earth's CMS (CMES: Earth's crust + upper mantle). Substituting the values into the equation we get: $\mathrm{RLTW}=(3 \cdot 7,3477 \cdot 1019$ ton $/ 4 \cdot 3,14 \cdot 4,5$ ton $/ \mathrm{m} 3=1574 \times 103 \mathrm{~m} \approx 1600 \mathrm{~km}$. The "eternal" tidal influence isolated the Main Oscillation System (MOS) with an average thickness of M0 $\approx 1600 \mathrm{~km}$ within the Earth's CMS. The MOS is characterized by rhythmic tectonic layering, fractal structure and, due to spatial variations in the density of the substance, variable lateral thickness. According to the tectonic layering mechanism, the depth of the Moho boundary in the CMS (the base of the Earth's crust) coincides with the uppermost M5 boundary of the MOS layering [2, 4, 8]. The thickness of the MOS allows the average density of the rocks $\rho$ CMES within the radius RLTW (for each case) to be calculated by the formula:

$$
\rho_{\mathrm{CMES}}=\rho_{\mathrm{L}} \cdot \mathrm{R}_{\mathrm{L}} / \mathrm{R}_{\mathrm{LTW}} \text {, }
$$

(4)

$\rho \mathrm{L}$ and $\mathrm{RL}$ - lunar average rocks density and radius, respectively.

\section{Gravity in spaces with different metrics}

From the basics of relativistic physics, by academician Okun' [6] it is known that the space-time continuum has a "flat" shape (a plane with two coordinate axes $\mathrm{X}$ and $\mathrm{Y})$. There is a theoretical interpretation of quantum physics, which describes the interaction of many different worlds with different metrics. Then the EarthMoon system, which is connected by a power "string", can be considered as one-dimensional. Earth sciences have not yet mastered the equations of quantum mechanics, so we would use the more accessible Newton laws, in particular, the law of universal gravitation.

The law of gravity: $\mathrm{F}=\gamma \mathrm{m} 1 \mathrm{~m} 2 / \mathrm{R} 2$ - acts in a 3dimensional world (No.3), measured in cubic meters (m3), and the force of mass interaction $(\gamma \mathrm{m} 1 \mathrm{~m} 2)$ for some reason depends only on the square of the distance $\mathrm{R} 2$ between them:

$$
\mathrm{F}^{\mathrm{No.3}}=\gamma \mathrm{m}_{1} \mathrm{~m}_{2} / \mathrm{R}^{(3-1)}=\gamma \mathrm{m}_{1} \mathrm{~m}_{2} / \mathrm{R}^{2} ;
$$

which is an occasion for reasoning. By analogy with the world No $=3$, in the world No.4 this dependence should be cubic (R3):

$$
\mathrm{F}^{\mathrm{No.4}}=\gamma \mathrm{m}_{1} \mathrm{~m}_{2} / \mathrm{R}^{3},
$$

in the world No.5 - biquadratic:

$$
\mathrm{F}^{\mathrm{No} .5}=\gamma \mathrm{m}_{1} \mathrm{~m}_{2} / \mathrm{R}^{4},
$$

etc. Even the most superficial analysis allows us to draw the following conclusion: an increase in the number of dimensions reduces the gravity force $\mathrm{F}$ and, accordingly, 
other forces of internal bonds in multidimensional systems, leading to weakening and a general decrease in the stability of such systems in proportion to the number of dimensions.

If we go in the opposite direction reducing the number of dimensions "convolution of space"): ... 2, 1, $0,-1 \ldots$ (integers), it is easy to make sure that as the number decreases, the gravitational force increase. So, for No. 2 (plane):

$$
\mathrm{F}^{\mathrm{No} .2}=\gamma \mathrm{m}_{1} \mathrm{~m}_{2} / \mathrm{R}^{1}
$$

- the dependence on R will be "just" inverse; for No. 1 (straight line or “string”):

$$
\mathrm{F}^{\mathrm{No.1}}=\gamma \mathrm{m}_{1} \mathrm{~m}_{2} / \mathrm{R}^{\mathbf{0}}
$$

Since $\mathrm{R} 0 \equiv 1$, any distance $(\mathrm{R}>0)$ would not affect FNo.1. If this is correct, then the dynamic parameters the propagation velocity $\mathrm{V}$ and propagation time $\mathrm{T}$ of the gravitational wave in world No.1 lose meaning, because: $\mathrm{R} 0=(\mathrm{VT}) 0 \equiv 1$ (for any values of $\mathrm{V}$ and $\mathrm{T}$ ).

In the world No. $0(\mathrm{R}=0)$, it is assumed the forcible introduction of two point masses at one point. But if there is room for only one point mass, such an experiment can lead to a sharp increase in pressure (density) at a point up to a "critical mass" and, possibly, cause an explosion at this point.

According to the rules of the "game of space convolution", the world No. 0 is followed by the world No. -1 :

$$
\mathrm{F}^{\text {No.-1 }}=\gamma \mathrm{m}_{1} \mathrm{~m}_{2} / \mathrm{R}^{-2}=\gamma \mathrm{m}_{1} \mathrm{~m}_{2} \cdot \mathrm{R}^{2} .
$$

In this space (No. -1), F grows in direct proportion to the square of the distance $\mathrm{R}$ between the masses: the further they diverge, the stronger they are pulled back (which resembles the work of hands with a gymnastic spring expander). Thus, in worlds with "negative No." with a shift of No. along the number line to the left of 0 , an additional compaction increases with strengthening bonds. All this resemble a description of the gravitational force in a hypothetical space object called the "black hole" [6].

Table 1. Estimated gravity (F) in worlds with metrics (No.) from 4 to -1.

\begin{tabular}{|c|c|l|}
\hline No. & *Equation for F & $\begin{array}{l}\text { Place in the hierarchy of } \\
\text { interactions }\end{array}$ \\
\hline 4 & $\mathrm{~F}^{4}=\gamma \mathrm{m}_{1} \mathrm{~m}_{2} / \mathrm{R}^{3}$ & $\begin{array}{l}\text { Probably, molecules of: } \mathrm{CH}_{4}, \\
\mathrm{SiCl}_{4}, \mathrm{SiH}_{4}, \text { etc. and ions: } \\
\mathrm{NH}_{4}^{+}, \mathrm{SO}_{4}{ }^{2+}, \mathrm{SiO}_{4}{ }^{4-} \text { etc. }\end{array}$ \\
\hline 3 & $\mathrm{~F}^{3}=\gamma \mathrm{m}_{1} \mathrm{~m}_{2} / \mathrm{R}^{2}$ & $\begin{array}{l}\text { Inertial interaction of the } \\
\text { masses in the "free fall" }\end{array}$ \\
\hline 2 & $\mathrm{~F}^{2}=\gamma \mathrm{m}_{1} \mathrm{~m}_{2} / \mathrm{R}^{1}$ & $\begin{array}{l}\text { Planets and stars in the ecliptic } \\
\text { planes of the corresponding } \\
\text { systems, galaxies, etc. }\end{array}$ \\
\hline 1 & $\mathrm{~F}^{1}=\gamma \mathrm{m}_{1} \mathrm{~m}_{2} / \mathrm{R}^{0}$ & $\begin{array}{l}\text { Along the lines of force } \\
\text { between the centers of mass of } \\
\text { binary systems: Earth-Moon, } \\
\text { etc. }\end{array}$ \\
\hline 0 & $\mathrm{~F}^{0}=\gamma \mathrm{m}_{1} \mathrm{~m}_{2} \cdot \mathrm{R}^{1}$ & From creating “critical \\
\hline
\end{tabular}

\begin{tabular}{|l|l|l|}
\hline & & $\begin{array}{l}\text { masses” to catastrophic } \\
\text { explosions. }\end{array}$ \\
\hline-1 & $\mathrm{~F}^{-1}=\gamma \mathrm{m}_{1} \mathrm{~m}_{2} \cdot \mathrm{R}^{2}$ & $\begin{array}{l}\text { Conditions for the birth of } \\
\text { objects of the "black hole" } \\
\text { type. }\end{array}$ \\
\hline
\end{tabular}

* Upper indice of the $\mathrm{F}$ denotes number of dimensions. of 0$)$ :

The law of gravity for measurements No. (both sides

No - integers (Table).

\section{Physics («quantum mechanism») of the solid tide waves}

Average thickness of the MOS layer is $\mathrm{M}_{\mathrm{MOS}} \approx 1600$ $\mathrm{km}$, the average elevation of the Earth's surface in the wave of a solid tide: $\mathrm{h} \approx 0.5 \mathrm{~m} \mathrm{[9]}$; therefore, the gravity perturbation can be considered as the elastic tension of the "string" - the radius of the tide wave (average radius $\mathrm{R}_{\mathrm{LTW}}=\mathrm{M}_{\mathrm{MOS}}$ ). The tensile strain of this "string" is $\Delta h=$ $\mathrm{h} / \mathrm{H}=0,5 \mathrm{~m} / 1600000 \mathrm{~m}=3,125 \cdot 10^{-7}$ per 1 meter of the "string". Assuming the average radius of an atom is: $\boldsymbol{r}_{\boldsymbol{a}} \approx$ $1 \AA=10^{-10} \mathrm{~m}$, then and one meter is a chain of $\mathrm{N} \approx 10^{10}$ atoms. Then the ratio of the elongation of the radius to the number of atoms in this chain gives the "tensile strain" of only one atom (or its electron cloud): $\Delta h_{a}=$ $3,125 \cdot 10^{-7} / 10^{10}=3,125 \cdot 10^{-17} \mathrm{~m}$. This number only differs from the amplitude of the "Nobel winning" wave $\left(\mathrm{A}=10^{-18} \mathrm{~m}\right)$ [1] by 1 order of magnitude. Since the most of chemical elements in the Earth's CMS occur as ions, and the ion radii are usually (often several times) less than atomic, the difference of 1 order of magnitude is easily "leveled". It is also known that due to gigantic pressures at depth the density of mantle matter differs significantly from that of a similar substance at the surface conditions (which is achieved via other types of densest atomic packs in mineral structures (see e.g., [10]). Thus, the order of magnitude of the amplitudes measured by LIGO and calculated (lunar tidal) gravitational waves practically coincide. Such a coincidence can be considered as a proof of the legitimacy of the EGM principle, which previously was only introduced as a postulate.

\section{Conclusions}

If the interaction in the Earth-Moon system proceeds as in World No.1 $\left(\mathrm{R}^{0} \equiv 1\right)$ and the distance from the source of the gravitational disturbance to the "receiver" does not matter, problematic questions are:

1) why is the cosmic catastrophe (merging of "black holes") assumed to be the source of the gravitational wave when it is believed that even a photon cannot escape from a "black hole?;

2) if this is indeed so, how the age of this merger $(\mathrm{T} \approx 1.3$ billion years ago) was determined? 
The coincidence in the sizes of the amplitude of gravitational waves, one of which was found on the LIGO interferometer, the other was obtained by simple calculations using the EGM principle (as part of a solid lunar tide wave) raises doubts about the advisability of continuing the interferometric program aimed at searching for new "sources of gravitational waves" in the distant Space. Instead of operating existing gravitationalwave detectors and building new (even more powerful and expensive), one should use the Earth for the same purposes, first having previously collected geophysical information about tidal wave effects in the Earth's CMS revised and reassessed.

This work was carried out as part of the State Assignment of the Geological Institute of the Kola Science Center of the Russian Academy of Sciences on the topic of research No. 0226-2019-0052.

\section{Reference}

1. B. Abbott, R. Abbott, T. Abbott at all., Phys. Rev. Lett. 116, 061102 (2016)

2. V. Il'chenko, Bull. of the Kola SC RAS 1, 173-178 (2012) [in Russian]

3. V. Il'chenko, Proceedings of the All-Russian meeting "Stress-strain state and seismicity of the lithosphere." (Novosibirsk Publ. House of the SB RAS, Branch "Geo", 49-53, 2003) [in Russian]

4. V. Il'chenko, Bull. of the Kola SC RAS 2(10) 53-62 (2018) [in Russian]

5. http://vladimirgorunovich.narod.ru/index/graviton_gravitino/0-99

6. L. B. Okun', ABC of Physics: A Very Brief Guide (World Scientific Publishing Co. Pte. Ltd., 2012)

7. A. Logunov, Priroda 4, 6-11 (2014) [in Russian] ISSN 0032-874X

8. V. Il'chenko. Proceedings of the XIII International Conference "Physicochemical and Petrophysical Studies in Earth Sciences", (Moscow, October 1-3, Borok, Oct. 4. M.: 2012), 109-112 (2011) [in Russian]

9. Yu. N. Avsyuk, Tidal forces and natural processes (M.: OIFZ RAS, 1996) [in Russian]

10. D. Pushcharovsky, Yu. Pushcharovsky, Soros Educational Journal 11, 111-119 (1998) [in Russian] 